\title{
Quantitative assessment of the effects of chitosan intervention on blood pressure control
}

This article was published in the following Dove Press journal:

Drug Design, Development and Therapy

\author{
Haohai Huangl,* \\ Ying Zou ${ }^{2,3, *}$ \\ Honggang $\mathrm{Chi}^{2}$ \\ 'Department of Clinical Pharmacy, \\ Dongguan Third People's Hospital, \\ Affiliated Dongguan Shilong People's \\ Hospital of Southern Medical \\ University, Dongguan, ${ }^{2}$ Department \\ of Traditional Chinese Medicine, \\ Scientific Research Platform, The \\ Second Clinical Medical College, \\ Guangdong Medical University, \\ Dongguan, ${ }^{3}$ Key Laboratory for \\ Medical Molecular Diagnostics of \\ Guangdong Province, Guangdong \\ Medical University, Dongguan, China \\ *These authors contributed equally \\ to this work
}

Correspondence: Honggang Chi Department of Traditional Chinese Medicine, The Second Clinical Medical College, Guangdong Medical University, No I, Xincheng Road of Songshan Lake Science and Technology Industry Park, Dongguan, Guangdong Province 523808, China

$\mathrm{Tel}+86$ I589969 588I

Fax +8676922896403

Email hgchil 168@I26.com
Background: Chitosan is a popular dietary fiber often used to reduce dietary fat absorption to control weight and blood lipids. However, its effects on blood pressure (BP) have not been fully elucidated. We evaluated the effects of chitosan administration on systolic blood pressure (SBP) and diastolic blood pressure (DBP) through a pooled analysis of available randomized controlled trials (RCTs).

Materials and methods: Electronic searches were conducted in Medline, Cochrane Library, Scopus, and EMBASE to identify relevant human placebo-control RCTs. Trials that reported $\mathrm{BP}$ changes from baseline to study endpoint in patients receiving treatment of chitosan were included for analysis. Weighted mean difference (WMD) and 95\% CIs were pooled using fixedeffects or random-effects models. Statistical heterogeneity, prespecified subgroup, publication bias, sensitivity analysis, and meta-regression assessments were also tested.

Results: Six hundred and seventeen participants from eight trials with 10 arms were included. Overall, chitosan administration did not significantly lower SBP (WMD: $-1.41 \mathrm{mmHg}, 95 \%$ CI: -3.29 to $0.47 ; P=0.14$ ) and DBP (WMD: $-0.61 \mathrm{mmHg}, 95 \% \mathrm{CI}:-1.75$ to $0.52 ; P=0.29$ ). However, our subgroup analyses indicated that chitosan consumption significantly reduced DBP in shorter-term ( $<12$ weeks) and higher-dose ( $>2.4 \mathrm{~g} /$ day $)$ arms. Funnel plots or Egger's tests analysis ( $P=0.36$ and 0.43 for SBP and DBP, respectively) demonstrated that there was no significant publication bias in this study.

Conclusion: This meta-analysis indicates that chitosan consumption significantly decreases DBP at higher dosage and in shorter-term interventions, while chitosan has no significant effects on SBP. However, these results should be interpreted cautiously because of the limited eligible RCTs included in this meta-analysis; further large-scale, well-designed RCTs on this topic are urgently needed.

Keywords: chitosan, hypertension, blood pressure, meta-analysis

\section{Introduction}

The prevalence of cardiovascular disease has been increasing globally. According to the World Health Organization, more than 17.5 million people die of cardiovascular disease per year, and this number is projected to rise to $>23.6$ million by $2030{ }^{1}$ Hypertension is an important risk factor for cardiovascular disease, myocardial infarction, stroke, and renal failure. ${ }^{2}$ Globally, two-thirds of stroke and one-half of ischemic heart disease events are attributable to poor blood pressure (BP) control, which contributes to $\sim 13 \%$ of all deaths and $4.5 \%$ of all disability-adjusted life years. ${ }^{3}$ The prevention and management of hypertension and related health complications are particularly important in reducing this burden. Accumulating evidence suggests that nutritional and lifestyle-based interventions and beneficial dietary supplements can assist in the treatment and control of hypertension and as adjuvants in pharmacologic therapies to reduce cardiovascular disease risk. ${ }^{4-6}$ 
Chitosan, a natural polysaccharide of $\beta$-1,4-linked glucosamine residues, is a biopolymer obtained primarily from the exoskeletons of crustaceans or from the cell walls of mushrooms. ${ }^{7}$ Dietary chitosan may help reduce the body weight and can lower blood lipids in both animal and human trials due to its chemical structure. ${ }^{8,9}$ However, the effects of chitosan on other cardiovascular risk factors, such as BP, have not been systematically assessed. Given the growing burden of hypertension, the suggested properties and benefits of dietary fiber consumption are an emergent area of research that warrants further scientific investigation. Evidence from experimental studies have indicated that administration of chitosan could prevent the development of hypertension in spontaneously hypertensive rats. ${ }^{10-12}$ Treatment of spontaneously hypertensive rats with water-soluble chitosan intervention strongly reduced the abnormally high BP and membrane thickness/lumen diameter ratio of abdominal aorta by suppressing the high levels of nuclear factor $\mathrm{c} 1$ of activated T cells (NFATc1) protein and even mRNA. ${ }^{11}$ Because of promising results in preclinical models, many clinical trials have been performed to determine the effect of chitosan intervention on the change of BP. ${ }^{13-16}$ However, these studies have a small sample size and convey mixed and inconclusive results. Therefore, given the increased statistical power afforded by meta-analysis and the enhanced precision of estimating effect sizes across several modest-sized trials, we conducted a meta-analysis of the evidence from available randomized controlled trials (RCTs) to evaluate the efficacy of chitosan administration on systolic and diastolic BP in humans. Our results inform whether chitosan supplements could be efficacious in preventing hypertension and cardiovascular disease.

\section{Materials and methods Data sources and searches}

We performed a literature search in Medline, EMBASE, and the Cochrane Central Register of Controlled Trials databases from January 1966 through March 2017 to identify RCTs examining the effects of chitosan on arterial BP. The following search terms were used: (Chitosan OR chitin) AND (blood pressure OR hypertension OR hypertensive OR diastolic blood pressure OR systolic blood pressure). Our search was confined to human studies without language restrictions. Furthermore, we also reviewed bibliographies of original research and previous reviews to complement the search.

\section{Selection of studies}

Potential articles were screened and selected based on our inclusion and exclusion criteria as follows: 1) adult participants (age $\geq 18$ years); 2) participants who used chitosan consumption in any form as an intervention (regardless of the type or application); 3) the study was an RCT in humans with either a parallel or crossover design; 4) trial reported effects of chitosan administration on BP, including SBP and/or DBP; and 5) presentation of sufficient information on baseline and at the end of study in both chitosan and control groups. Studies were excluded if they 1) did not report the dose of chitosan consumption; 2) had a consumption duration of $<2$ weeks; and 3) were reported in abstract form or letters only.

\section{Quality control}

We utilized the Cochrane Collaboration's tool to assess the bias of included trials. ${ }^{17}$ Random sequence generation, allocation concealment, blinding of participants and personnel, blinding of outcome assessment, incomplete outcome data, selective reporting, and other sources of bias were judged to be high, low, or unclear in each of the studies included. A judgment of " $L$ " indicated low risk of bias, while " $\mathrm{H}$ " indicated high risk of bias. Labeling an item as " $U$ " indicated an unclear or unknown risk of bias.

\section{Data extraction}

From each study, we abstracted the first author's name, year of publication, study design (crossover or parallel; single blind or double blind), intervention duration, form and dosage of chitosan intake, number of patients involved, country of origin, and the characteristics of the participants (including age, gender, baseline values for body mass index, and health status). If the trials included in our analysis had multiple intervention groups, we grouped together all the experimental groups and compared them with the control group. Disagreements were also resolved through discussion and consensus.

\section{Data synthesis and analysis}

We calculated weighted mean difference (WMD) and 95\% CIs between the intervention and control groups for continuous outcomes. Heterogeneity across the studies was tested using the $I^{2}$ statistic, and an $I^{2}$ value of $>50 \%$ indicated significant heterogeneity. Primary analyses were conducted with a fixed-effects model, and secondary confirmatory analyses were conducted with a random-effects model if there was significant heterogeneity. ${ }^{18}$ To examine the effects of factors on the primary outcomes, several previously defined subgroup analyses were considered according to the intervention duration, study design, and chitosan dose. In order to evaluate the influence of each study on the overall effect size, 
sensitivity analysis was conducted by removing one study at a time or changing the effects models independently. Presence of publication bias in the meta-analysis was assessed by visually inspecting a funnel plot and was also evaluated by using the Egger's test. ${ }^{19}$ All tests were two-tailed and a $P$-value of $<0.05$ was considered statistically significant for all analyses. All statistical analyses were performed using the STATA program (Version 12.0; StataCorp LP, College Station, TX, USA).

\section{Meta-regression analysis}

To evaluate the association between calculated net changes in outcomes and putative confounders, the effects of dosage and duration of chitosan administration on BP were determined by a restricted maximum likelihood-based meta-regression analysis. ${ }^{20}$

\section{Results}

\section{Retrieved data and characteristics of the trials}

In summary, eight RCTs (equivalent to 10 treatment arms) met our inclusion criteria and were preferred for the final pooled analysis. ${ }^{14-16,21-25}$ Figure 1 shows the number of studies assessed and excluded through the stages of the meta-analysis. A summary of the basic characteristics of the included trials and participants is given in Table 1. These trials were published between 1999 and 2005. Two of the trials were conducted in Finland; ${ }^{16,22}$ the others were conducted in Japan, ${ }^{15} \mathrm{UK},{ }^{14}$ Singapore,${ }^{21}$ Australia,${ }^{23}$ Italy,${ }^{24}$ and Poland. ${ }^{25}$ The number of participants included in these studies ranged from 30 to 250 (total 617). The age of participants among all trials ranged from 22 to 80 years. Crossover design was reported in two studies, ${ }^{16,22}$ and six trials reported parallel design. ${ }^{14,15,21,23-25}$ The chitosan administration method varied among the trials, with six studies reporting use of chitosan capsulation, ${ }^{14,15,21,23-25}$ and two studies reporting use of microcrystalline chitosan. ${ }^{16,22}$ A range of doses from 1 to $4.5 \mathrm{~g} /$ day of chitosan were administered in these included trials, with a median of $2.41 \mathrm{~g} /$ day. The duration of the chitosan intervention varied from 4 to 24 weeks (median: 12.4 weeks). The body mass index values of patients at baseline ranged from 22.9 to $35.5 \mathrm{~kg} / \mathrm{m}^{2}$. Selected studies were performed in subjects with overweight or obesity, hypercholesterolemia, and prehypertension (defined as systolic BP ranging from 120 to $139 \mathrm{mmHg}$ and/or diastolic BP ranging from 80 to $89 \mathrm{mmHg}$ ). The baseline levels of BP were generally matched between the chitosan and control groups in the included studies. An adequate randomized sequence was generated in six trials, ${ }^{14-16,22,23,25}$ and two selected studies did not provide sufficient data about random sequence generation. ${ }^{21,24}$ The details of the risk-of-bias analysis are shown in Table 2.

Potentially relevant titles and abstracts identified through electronic search $(n=142)$ : (filters: human subjects; RCTs; up to May 2017)

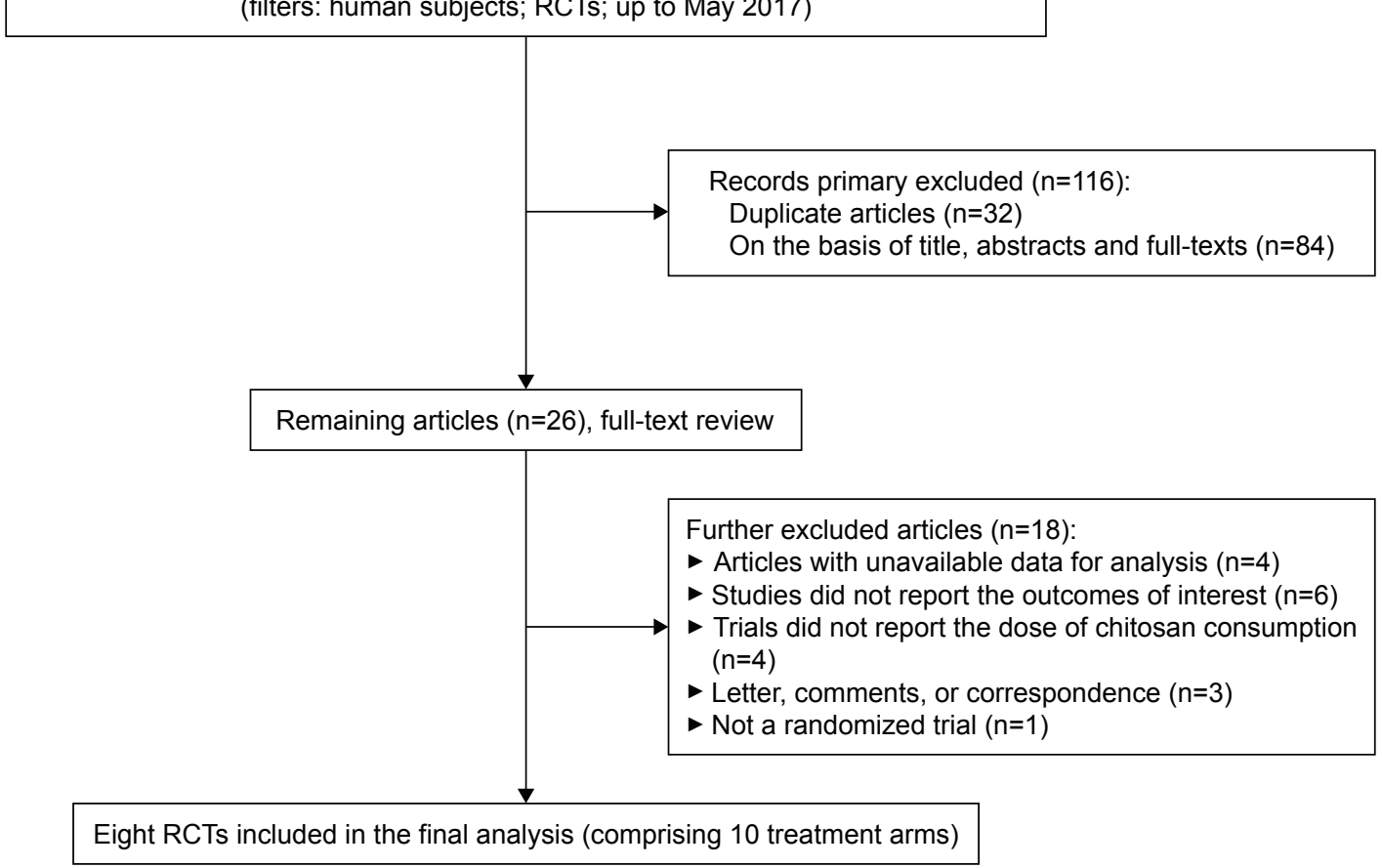

Figure I Flow diagram for identification of relevant studies. 


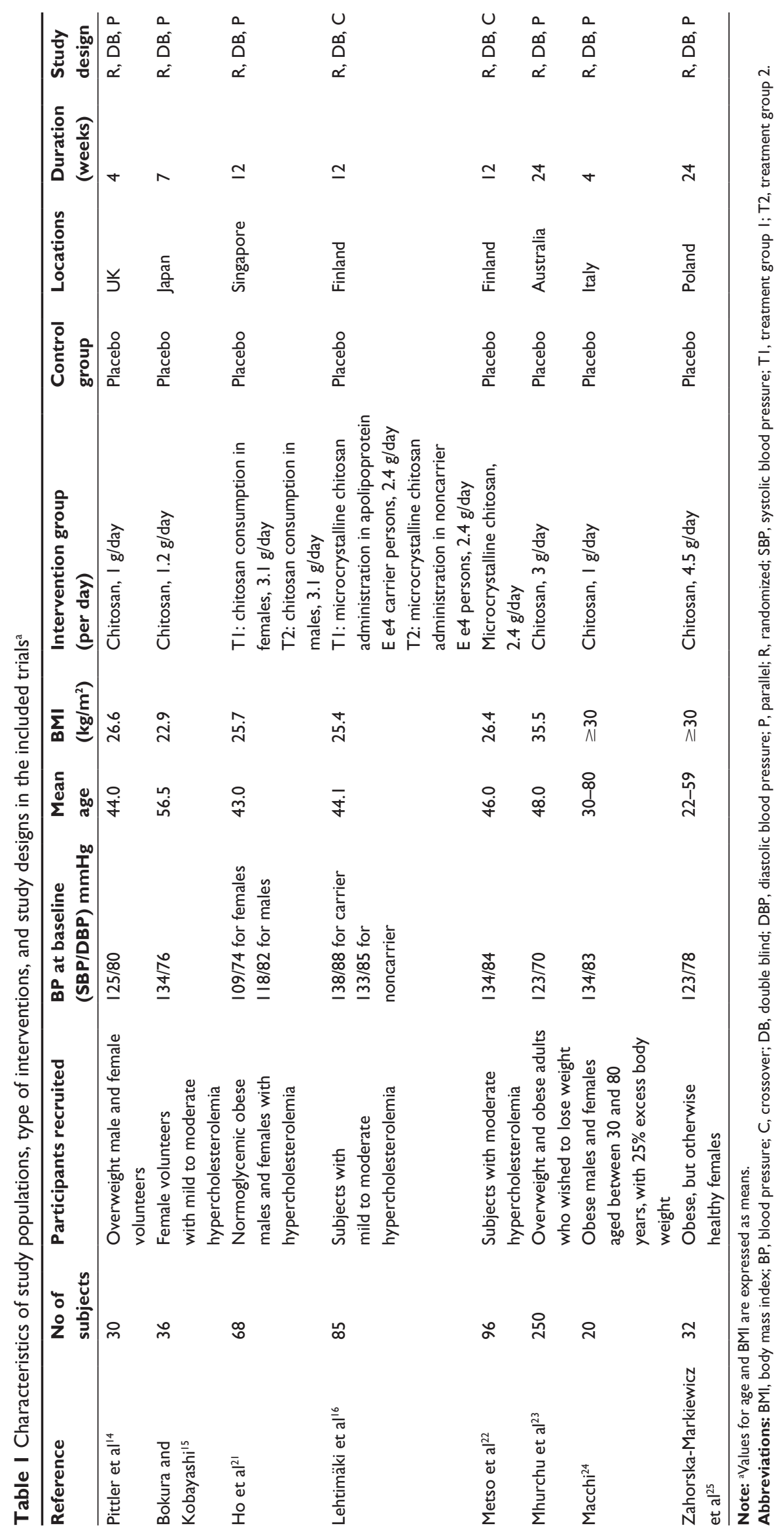


Table 2 Quality assessment of the included studies ${ }^{\mathrm{a}}$

\begin{tabular}{|c|c|c|c|c|c|c|c|c|}
\hline Study ID & Year & $\begin{array}{l}\text { Random } \\
\text { sequence } \\
\text { generation }\end{array}$ & $\begin{array}{l}\text { Allocation } \\
\text { concealment }\end{array}$ & $\begin{array}{l}\text { Blinding of } \\
\text { participants } \\
\text { and personnel }\end{array}$ & $\begin{array}{l}\text { Blinding of } \\
\text { outcome } \\
\text { assessment }\end{array}$ & $\begin{array}{l}\text { Incomplete } \\
\text { outcome } \\
\text { data }\end{array}$ & $\begin{array}{l}\text { Selective } \\
\text { reporting }\end{array}$ & $\begin{array}{l}\text { Other } \\
\text { sources } \\
\text { of bias }\end{array}$ \\
\hline Pittler et al $^{14}$ & 1999 & L & L & L & L & L & L & L \\
\hline Bokura and Kobayashi ${ }^{15}$ & 2003 & L & L & L & L & L & L & L \\
\hline Ho et $\mathrm{al}^{21}$ & 2001 & $U$ & L & L & L & L & L & L \\
\hline Lehtimäki et al' ${ }^{16}$ & 2005 & $\mathrm{~L}$ & L & L & L & L & L & L \\
\hline Metso et $\mathrm{a}^{22}$ & 2003 & L & L & L & L & L & L & L \\
\hline Mhurchu et $\mathrm{al}^{23}$ & 2004 & L & L & L & L & L & L & L \\
\hline Macchi $^{24}$ & 1996 & $\cup$ & L & L & L & L & L & L \\
\hline Zahorska-Markiewicz et al ${ }^{25}$ & 2002 & $\mathrm{~L}$ & L & L & $\mathrm{L}$ & L & L & L \\
\hline
\end{tabular}

Note: an all eligible studies, criteria defined for quality assessment are based on the Cochrane guidelines.

Abbreviations: $L$, low risk of bias; $U$, unclear or unrevealed risk of bias.

\section{Effects of chitosan administration on SBP and DBP}

The results for BP were reported in eight RCTs (10 treatment arms) representing 617 participants. As shown in Figure 2, chitosan intervention did not significantly influence SBP (WMD: $-1.41 \mathrm{mmHg}, 95 \% \mathrm{CI}:-3.29$ to $0.47 ; P=0.14$ ), without significant heterogeneity $\left(I^{2}=0 \%\right)$. No significant decrease was detected for the effect of chitosan on DBP (WMD: $-0.61 \mathrm{mmHg}, 95 \% \mathrm{CI}:-1.75$ to $0.52 ; P=0.29 ; I^{2}=25 \%$ ). The results of a fixed-effects model in this meta-analysis were exactly the same as those of a random-effects model.

\section{Subgroup analyses and sensitivity analyses}

We conducted subgroup analyses to explore the effects of study design, chitosan dose, and intervention duration on the overall effects of chitosan on both SBP and DBP. The chitosan dose was categorized as either a lower dose $(<2.4 \mathrm{~g}$ /day) or a higher dose $(\geq 2.4 \mathrm{~g} /$ day $)$. The duration of chitosan intervention was divided into a longer-term subgroup ( $\geq 12$ weeks) and a shorter-term subgroup ( $<12$ weeks). In a subgroup analysis stratified by study design, the administration of chitosan did not significantly decrease the levels of SBP and DBP both in parallel and crossover design groups. Similarly, the subgroup analyses indicated that differences in chitosan dose and intervention duration did not appear to significantly influence pooled mean differences in SBP concentrations. However, a significant reduction in DBP was observed in higher-dose (WMD: $-3.50 \mathrm{mmHg}, 95 \%$ CI: -6.40 to $\left.-0.59 ; P=0.02 ; I^{2}=26 \%\right)$ and shorter-term (WMD: $-4.47 \mathrm{mmHg}, 95 \% \mathrm{CI}:-7.66$ to $-1.27 ; P=0.006$; $I^{2}=11 \%$ ) trials. The summary of the subgroup analysis results is presented in Table 3 .

In addition, sensitivity analysis showed that the pooled effects of chitosan on BP did not substantially change after changing the effects model (Table 3). Further exclusion was conducted by omitting any single study that did not materially alter the overall combined WMD, which adds robustness to our main finding.

\section{Meta-regression analysis}

We conducted univariate meta-regression analyses for the following potential moderator variables: dosage of chitosan intervention and duration of chitosan administration. The meta-regression results did not indicate any significant association between duration of administration and impact of chitosan on SBP (coefficient: $-0.07,95 \%$ CI: -0.29 to $0.27 ; P=0.949$ ) and DBP (coefficient: $1.75,95 \% \mathrm{CI}:-0.09$ to $0.68 ; P=0.117)$. In addition, there was also no association between the dose of chitosan consumed per day and respective changes in plasma SBP (coefficient: $-0.19,95 \%$ CI: -2.19 to $1.86 ; P=0.854$ ) and DBP (coefficient: $0.77,95 \%$ CI: -1.39 to $2.80 ; P=0.462$ ).

\section{The risk of bias and publication bias}

Potential publication bias was examined by analyzing funnel plots and Egger's tests. In all trials, the funnel plots were symmetrical (Figure 3). Results from Begg's tests also did not indicate evidence of publication bias (for SBP, $P=0.36$; for DBP, $P=1.43$ ).

\section{Discussion}

The number of people with hypertension is estimated to grow to more than 1.5 billion by 2025 , adding to the growing economic and social burden of the disease. Dietary recommendations for patients with hypertension include reduction of sodium intake, moderation of alcohol consumption, and the Dietary Approaches to Stop Hypertension eating plan recommended by the American Heart Association. ${ }^{26}$ We comprehensively and systematically reviewed the current available RCTs that evaluate the effects of chitosan administration on SBP and DBP. In the final analysis, a total of eight articles 

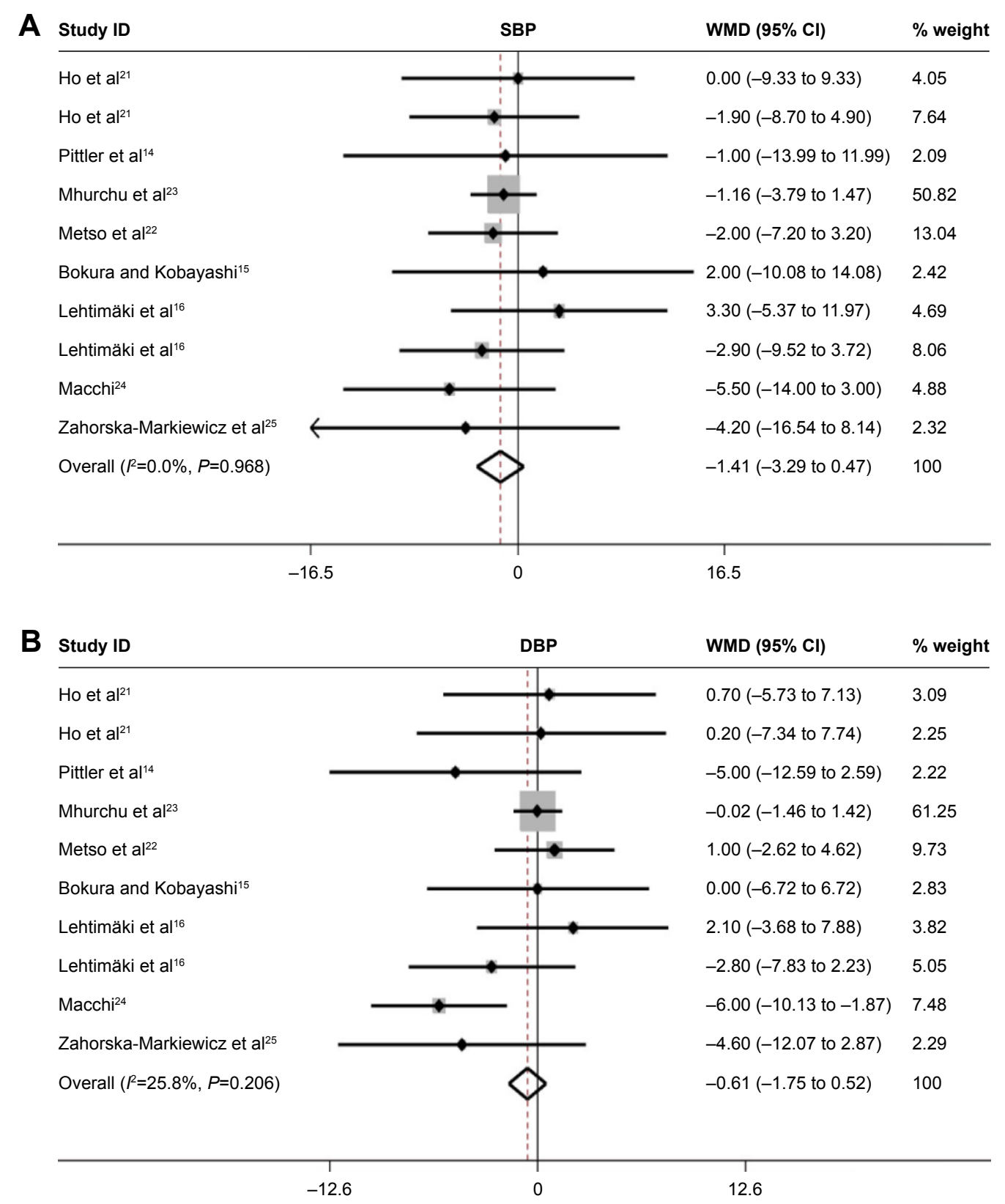

Figure 2 Net changes in systolic blood pressure (A) and diastolic blood pressure (B) in randomized trials of chitosan supplementation.

Notes: The area of each square is proportional to the study weight in the analysis. Horizontal lines represent $95 \%$ Cls. Diamonds represent pooled estimates.

Abbreviations: DBP, diastolic blood pressure; SBP, systolic blood pressure; WMD, weighted mean difference.

with 10 treatment arms were included for pooling. Findings from this study demonstrate that the overall effects of chitosan consumption did not significantly decrease the levels of SBP and DBP in humans. Additional subgroup analyses of chitosan dose and study duration did not significantly affect the pooled results of the effect of chitosan on SBP. However, the results of subgroup analysis based on the chitosan dose and study duration further revealed that daily ingestion of chitosan significantly decreased DBP values in higher-dose ( $\geq 2.4 \mathrm{~g} /$ day, $P=0.02$ ) and shorter-term ( $<12$ weeks, $P=0.006$ ) groups. Our meta-regression analysis did not indicate any significant association between duration of administration/ dose of chitosan and impact of chitosan on SBP and DBP.

The main finding in this study seems to contradict the previous meta-analysis, which was performed to appraise the impact of the administration of chitosan on the change of BP. A systematic review conducted by Jull et $\mathrm{al}^{27}$ provided conclusive evidence that chitosan significantly lowered the levels of SBP (WMD: $-6 \mathrm{mmHg}, 95 \% \mathrm{CI}:-7$ to -5 ; $P<0.00001$ ) and DBP (WMD: $-3 \mathrm{mmHg}, 95 \% \mathrm{CI}:-4$ to -2 ; 
Table 3 Subgroup and sensitivity analyses of BP stratified by previously defined study characteristics

\begin{tabular}{|c|c|c|c|c|c|c|c|c|}
\hline \multirow[t]{2}{*}{ Variables } & \multirow[t]{2}{*}{ Arms } & \multicolumn{3}{|l|}{ SBP $(\mathbf{m m H g})$} & \multirow[t]{2}{*}{ Arms } & \multicolumn{3}{|l|}{ DBP (mmHg) } \\
\hline & & WMD (95\% Cl) & $P$-value & $I^{2}(\%)$ & & WMD $(95 \% \mathrm{Cl})$ & $P$-value & $I^{2}(\%)$ \\
\hline \multicolumn{9}{|l|}{ Subgroup analyses } \\
\hline \multicolumn{9}{|l|}{ Intervention duration } \\
\hline Shorter term $(<12$ weeks $)$ & 3 & $-2.57(-8.70$ to 3.56$)$ & $0.4 \mathrm{I}$ & 0 & 3 & $-4.47(-7.66 \text { to }-1.27)^{\mathrm{a}}$ & 0.006 & 11 \\
\hline Longer term ( $\geq 12$ weeks) & 7 & $-1.29(-3.27$ to 0.68$)$ & 0.20 & 0 & 7 & $-0.06(-1.27$ to 1.15$)$ & 0.92 & 0 \\
\hline \multicolumn{9}{|l|}{ Dose } \\
\hline$\leq 2.4 \mathrm{~g} /$ day & 6 & -1.11 (-3.20 to 0.97$)$ & 0.30 & 0 & 6 & $-0.10(-1.33$ to 1.12$)$ & 0.87 & 0 \\
\hline$>2.4 \mathrm{~g} /$ day & 4 & $-2.7 \mid(-7.03$ to I.62) & 0.22 & 0 & 4 & $-3.50(-6.40 \text { to }-0.59)^{a}$ & 0.02 & 26 \\
\hline \multicolumn{9}{|l|}{ Study design } \\
\hline Parallel & 7 & $-1.45(-3.63$ to 0.73$)$ & 0.19 & 0 & 7 & $-0.80(-2.05$ to 0.45$)$ & 0.21 & 38 \\
\hline Crossover & 3 & $-1.32(-5.02$ to 2.38$)$ & 0.49 & 0 & 3 & 0.19 (-2.43 to 2.82$)$ & 0.88 & 0 \\
\hline \multicolumn{9}{|l|}{ Sensitivity analysis } \\
\hline Fixed-effects models & 10 & $-1.4 \mid(-3.29$ to 0.47$)$ & 0.14 & 0 & 10 & -0.61 ( -1.75 to 0.52$)$ & 0.29 & 26 \\
\hline Random-effects models & 10 & $-|.4|(-3.29$ to 0.47$)$ & 0.14 & 0 & 10 & $-1.06(-2.75$ to 0.62$)$ & 0.22 & 26 \\
\hline
\end{tabular}

Note: andicates a significant result.

Abbreviations: DBP, diastolic blood pressure; SBP, systolic blood pressure; WMD, weighted mean difference.

$P<0.00001)$. Heterogeneity was noted for these outcomes (for SBP, $I^{2}=78.5 \%$; for DBP, $I^{2}=86.3 \%$ ). There are seven trials included in the previous review, including four of the studies described here. Our analysis did not include three RCTs that were included in the previous meta-analysis because these RCTs did not report the dose of chitosan consumption, which did not meet our stringent inclusion criteria. This meta-analysis has several strengths. In an attempt to produce robust results, we prestated rigorous inclusion criteria and included only those RCTs that clearly stated the enrollment of patients ingesting chitosan formulations and that specifically referred to BP change. Other strengths of the study were as follows: possible absence of publication bias in trials with chitosan administration by visual inspection of funnel plots; the low heterogeneity observed in the majority of forest plots; and in most trials with chitosan administration, the subjects were not taking BP-lowering drugs, which makes the conclusions of the analysis more consistent. To investigate the effects of chitosan on various parameters among these studies, previously defined subgroup analyses, such as intervention duration, chitosan dose, and study design, were also performed. Moreover, exclusion of any single study and sensitivity analyses based on various effects models did not materially alter the pooled results, which supported the robustness of our results. Finally, there was no evidence of publication bias of the studies by using Egger's regression test and the funnel plots.

According to the National Health and Nutrition Examination Survey study, isolated systolic hypertension (systolic $\geq 140 \mathrm{mmHg}$ with diastolic $<90 \mathrm{mmHg}$ ) was present in $65 \%$ of all hypertensives $>60$ years of age, whether male or female. Besides, diastolic hypertension was more prevalent among patients $<50$ years of age, but for those older than 50 years, systolic hypertension was a better risk predictor of
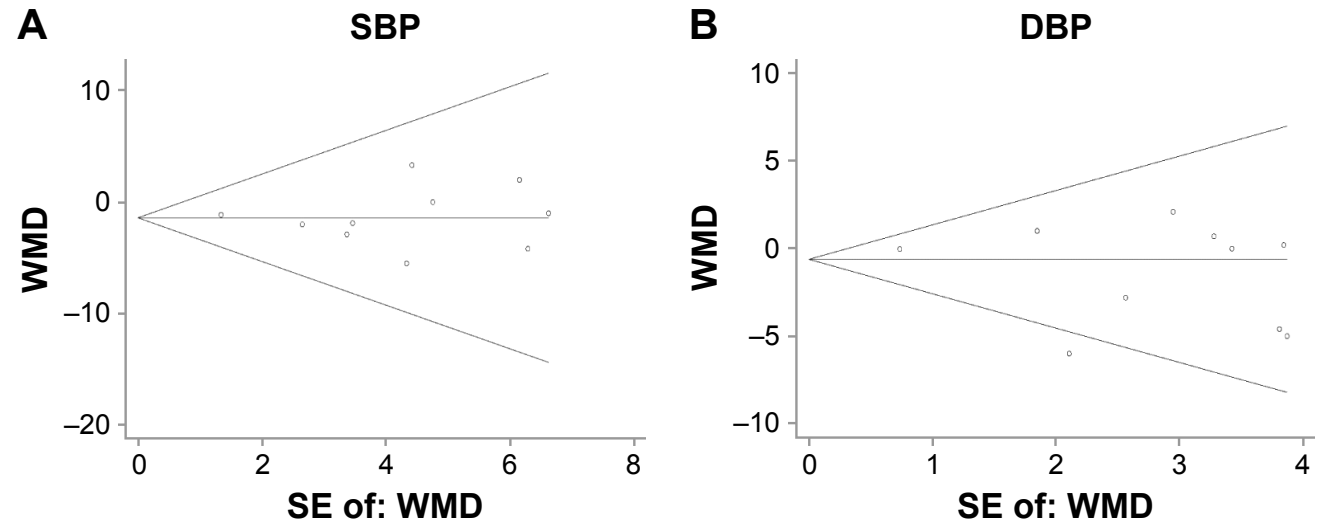

Figure 3 Funnel plots with pseudo $95 \%$ Cls for publication bias of systolic blood pressure (A) and diastolic blood pressure (B).

Abbreviations: DBP, diastolic blood pressure; SBP, systolic blood pressure; SE, standard error; WMD, weighted mean difference. 
cardiovascular events. ${ }^{28}$ In our subgroup analysis, the pooled analysis further demonstrated that a significant reduction in DBP was observed in subjects who consumed $>2.4 \mathrm{~g}$ of chitosan daily. Perhaps once deacetylated, chitosan can act as a cationic polysaccharide in the intestinal tract. Positively charged amino groups on chitosan bind to negatively charged molecules such as fatty acids, lipids, and bile acids, after which they are excreted from the body rather than being systemically absorbed or reabsorbed. ${ }^{29}$ Therefore, the concentration of chitosan in the plasma of subjects under treatment with a lower dose might not be sufficient to regulate DBP. Regarding the subgroup analysis by intervention duration, a similar significant change in DBP was observed in subjects who consumed chitosan for $<12$ weeks. Most notably, these results are inconclusive because of the limited eligible RCTs included in these subgroups; further adequately powered studies are needed.

Although this meta-analysis provides useful information for clinicians and researchers alike, several important limitations and gaps are worth noting. First, none of the included trials was designed to investigate the effect of chitosan on BP, and BP measurements were not the primary endpoints. The null findings of the secondary outcomes may not have always been published. Secondly, although clear inclusion and exclusion criteria were made, significant variations still existed among different study designs, dose of chitosan consumption, and patient's daily diet, which produced a certain bias to the research results. Another inherent limitation of meta-analysis is the limitation of data in the original published articles and lack of access to the individual patientlevel data which would have been particularly helpful for our subgroup analyses and to explain the heterogeneity among studies. Finally, given the lack of data in each trial, we did not adjust our analyses for compliance to assigned therapy.

\section{Conclusion}

The current evidence suggests that daily chitosan consumption significantly decreases DBP in higher-dosage $(>2.4 \mathrm{~g} /$ day $)$ and shorter-term $(<12$ weeks) interventions, while chitosan has no significant effects on SBP. However, these results are inconclusive and should be interpreted cautiously because of the limited number of RCTs regarding the effect of higher dose of chitosan and shorter-term interventions on the measure of BP. Further large-scale, well-designed RCTs on this topic are urgently needed.

\section{Acknowledgments}

This work was supported by the National Natural Science Foundation of China under grant no 81603592, the Natural
Science Foundation of Guangdong Province under grant no 2016A030310356, and the Social Development Project of Dongguan City under grant no 2014108101054.

\section{Disclosure}

The authors report no conflicts of interest in this work.

\section{References}

1. Smith SC Jr, Collins A, Ferrari R, et al. Our time: a call to save preventable death from cardiovascular disease (heart disease and stroke). Circulation. 2012;126(23):2769-2775.

2. Ong KL, Cheung BM, Man YB, Lau CP, Lam KS. Prevalence, awareness, treatment, and control of hypertension among United States adults 1999-2004. Hypertension. 2007;49(1):69-75.

3. Benjamin EJ, Blaha MJ, Chiuve SE, et al. Heart disease and stroke statistics-2017 update: a report from the American Heart Association. Circulation. 2017;135(10):e146-e603.

4. Peng YG, Li W, Wen XX, Li Y, Hu JH, Zhao LC. Effects of salt substitutes on blood pressure: a meta-analysis of randomized controlled trials. Am J Clin Nutr. 2014;100(6):1448-1454.

5. Desch S, Schmidt J, Kobler D, et al. Effect of cocoa products on blood pressure: systematic review and meta-analysis. Am J Hypertens. 2010;23(1):97-103.

6. Serban MC, Sahebkar A, Zanchetti A, et al; Lipid and Blood Pressure Meta-analysis Collaboration (LBPMC) Group. Effects of quercetin on blood pressure: a systematic review and meta-analysis of randomized controlled trials. J Am Heart Assoc. 2016;5(7):e002713.

7. Bueter CL, Specht CA, Levitz SM. Innate sensing of chitin and chitosan. PLoS Pathogens. 2013;9(1):e1003080.

8. Pittler MH, Ernst E. Dietary supplements for body-weight reduction: a systematic review. Am J Clin Nutr. 2004;79(4):529-536.

9. Baker WL, Tercius A, Anglade M, White CM, Coleman CI. A metaanalysis evaluating the impact of chitosan on serum lipids in hypercholesterolemic patients. Ann Nutr Metab. 2009;55(4):368-374.

10. Park SH, Dutta NK, Baek MW, et al. NaCl plus chitosan as a dietary salt to prevent the development of hypertension in spontaneously hypertensive rats. $J$ Vet Sci. 2009;10(2):141-146.

11. Du M, Kou L, Li S. Water-soluble chitosan regulates vascular remodeling in hypertension via NFATc1. Eur Heart J Suppl. 2016; 18(Suppl F):F38.

12. Hong SP, Kim MH, Oh SW, Han CK, Kim YH. ACE inhibitory and antihypertensive effect of chitosan oligosaccharides in SHR. Korean J Food Sci Technol. 1998;30(1):1476-1479.

13. Allaert FA. Effect of $\mathrm{NaCl}+\mathrm{Chitosan} 3 \%$ vs. $\mathrm{NaCl}$ on high blood pressure parameters of healthy volunteers with prehypertension. Minerva Cardioangiol. 2017;65(6):563-576.

14. Pittler MH, Abbot NC, Harkness EF, Ernst E. Randomized, doubleblind trial of chitosan for body weight reduction. Eur J Clin Nutr. 1999; 53(5):379-381.

15. Bokura H, Kobayashi S. Chitosan decreases total cholesterol in women: a randomized, double-blind, placebo-controlled trial. Eur J Clin Nutr. 2003;57(5):721-725.

16. Lehtimäki T, Metso S, Ylitalo R, et al. Microcrystalline chitosan is ineffective to decrease plasma lipids in both apolipoprotein E epsilon 4 carriers and non-carriers: a long-term placebo-controlled trial in hypercholesterolaemic volunteers. Basic Clin Pharmacol Toxicol. 2005; 97(2):98-103.

17. Higgins JP, Green S. Cochrane Handbook for System-atic Reviews of Interventions Version 5.1.0. (updated March 2011). Available from: http://handbook.cochrane.org/. Accessed August 02, 2017.

18. Higgins JP, Thompson SG, Deeks JJ, Altman DG. Measuring inconsistency in meta-analyses. BMJ. 2003;327(7414):557-560.

19. Egger M, Davey Smith G, Schneider M, Minder C. Bias in meta-analysis detected by a simple, graphical test. BMJ. 1997;315(7109):629-634.

20. Thompson SG, Higgins JP. How should meta-regression analyses be undertaken and interpreted? Stat Med. 2002;21(11):1559-1573. 
21. Ho SC, Tai ES, Eng PH, Tan CE, Fok AC. In the absence of dietary surveillance, chitosan does not reduce plasma lipids or obesity in hypercholesterolaemic obese Asian subjects. Singapore Medical J. 2001; 42(1):6-10.

22. Metso S, Ylitalo R, Nikkila M, Wuolijoki E, Ylitalo P, Lehtimäki T. The effect of long-term microcrystalline chitosan therapy on plasma lipids and glucose concentrations in subjects with increased plasma total cholesterol: a randomised placebo-controlled double-blind crossover trial in healthy men and women. Eur J Clin Pharmacol. 2003; 59(10):741-746.

23. Mhurchu CN, Poppitt SD, McGill AT, et al. The effect of the dietary supplement, Chitosan, on body weight: a randomised controlled trial in 250 overweight and obese adults. Int J Obes Relat Metab Disord. 2004;28(9):1149-1156.

24. Macchi G. A new approach to the treatment of obesity: chitosan's effects on body weight reduction and plasma cholesterol's levels. Acta Toxicol Ther. 1996;17:301-320.
25. Zahorska-Markiewicz B, Krotkiewski M, Olszanecka-Glinianowicz M, Zurakowski A. [Effect of chitosan in complex management of obesity]. Pol Merkur Lekarski. 2002;13(74):129-132. Polish.

26. Appel LJ, Brands MW, Daniels SR, Karanja N, Elmer PJ, Sacks FM; American Heart Association. Dietary approaches to prevent and treat hypertension: a scientific statement from the American Heart Association. Hypertension. 2006;47(2):296-308.

27. Jull AB, Ni Mhurchu C, Bennett DA, Dunshea-Mooij CA, Rodgers A. Chitosan for overweight or obesity. Cochrane Database Syst Rev. 2008;3:CD003892.

28. Taku K, Lin N, Cai D, et al. Effects of soy isoflavone extract supplements on blood pressure in adult humans: systematic review and metaanalysis of randomized placebo-controlled trials. J Hypertens. 2010; 28(10):1971-1982.

29. Tapola NS, Lyyra ML, Kolehmainen RM, Sarkkinen ES, Schauss AG Safety aspects and cholesterol-lowering efficacy of chitosan tablets. J Am Coll Nutr. 2008;27(1):22-30.

\section{Publish your work in this journal}

Drug Design, Development and Therapy is an international, peerreviewed open-access journal that spans the spectrum of drug design and development through to clinical applications. Clinical outcomes, patient safety, and programs for the development and effective, safe, and sustained use of medicines are the features of the journal, which has also been accepted for indexing on PubMed Central. The manuscript management system is completely online and includes a very quick and fair peer-review system, which is all easy to use. Visit http://www.dovepress.com/testimonials.php to read real quotes from published authors.

Submit your manuscript here: http://www.dovepress.com/drug-design-development-and-therapy-journal 